\title{
Basisorganisierung verändert die politische Landschaft
}

Claudia Kratzsch, Robert Maruschke/Basisorganisierung verändert die politische Landschaft

Kommentare von: Workers Center München, Kotti\&Co Berlin, Rothe Ecke Kassel

Replik von: Robert Maruschke

\author{
Claudia Kratzsch
}

Robert Maruschke

\begin{abstract}
Aktuelle Debatten aus dem Kontext linker sozialer Bewegungen betonen Organisationsfragen. Dieser Essay kritisiert die übliche These, der zufolge dynamische soziale Bewegungen und feste Organisierung einander widersprechen. Stattdessen plädieren die Autor_innen für ein miteinander verzahntes Verständnis von Bewegungen und Organisation - angelehnt an das Konzept des transformativen Organizings. Sie skizzieren einige wichtige Aspekte linker Basisorganisierung und fordern eine ehrliche Bestandsaufnahme ein. Ermöglichen es die vorherrschenden Strategien sozialer Bewegungen unterschiedlichen Menschen, sich in soziale Konflikte einzubringenen, diese zu gestalten und eine gesellschaftliche Vision abseits tagespolitischer Zumutungen zu entwerfen? Diese Fragen bilden den Ausgangspunkt für eine Debatte mit mehreren Basis-Initiativen.
\end{abstract}

An english abstract can be found at the end of the document.

Strategiedebatten sollten für soziale Bewegungen und ihre Initiativen, Gruppen oder Organisationen zur Routine gehören. Die eigene Herangehensweise regelmäßig zu überprüfen, ist ein mitunter unangenehmes Unterfangen, weil man sich gegebenenfalls eigene Fehleinschätzungen eingestehen und diese korrigieren muss. Eventuell erweist sich ein großer Teil der geleisteten Arbeit als verlorene Liebesmüh was im ersten Moment schwer auszuhalten ist, langfristig aber vor ähnlichen Fehleinschätzungen bewahren kann. Strategiedebatten können aber auch zunächst unauffällige Aspekte der politischen Arbeit ins Zentrum der Aufmerksamkeit rücken oder bisher wenig beachtete Kraftanstrengungen zur Würdigung verhelfen.

In der mit diesem Artikel eröffneten Debatte wollen wir unterschiedliche Erfahrungen aus den letzten Jahren zusammenführen und Interessierten zugänglich machen. Nur allzu oft versauern die Ergebnisse produktiver Selbstreflektionen in Plenumsordnern oder auf Festplatten und Emailservern, wenn sie es denn überhaupt bis dahin schaffen. Unsere Perspektiven auf die Lage linker außerparlamentarischer Politik haben uns zu der Einschätzung gebracht, dass den Zumutungen des Alltags politisch nur mit vielen neuen Projekten der Basisorganisierung beizukommen ist. 


\section{Basisorganisierung und Bewegung - ein Gegensatz?}

Basisorganisierung ist ein Begriff, der durch seine Unbestimmtheit Gefahr läuft, produktive Kontroversen zu verhindern. Je nach Blickwinkel und politischer Prägung kann Basisorganisierung vieles bedeuten und von (fast) allen für gut und richtig gefunden werden. Die sehr konkrete und praktische Frage, durch welche Organisations- und Aktionsformen linke Bewegungen die selbstgesteckten Ziele erreichen können, kann mit einer unbestimmten Vorstellung von Basisorganisierung nur unzureichend beantwortet werden. Durch unseren Text und die nachfolgende Debatte wollen wir an anderer Stelle begonnene Strategiegespräche vertiefen[1]. In den letzten Jahren haben wir die Konturen unseres Verständnisses von Basisorganisierung geschärft, vieles praktisch ausprobiert und einige Diskussionen mit anderen Aktiven geführt. Dabei sind wir auf Konzepte des Organizings gestoßen, die oft als Modestrategien abgetan werden. Es wird argumentiert, dass diese Konzepte linken sozialen Bewegungen keine neuen Anregungen für gegenwärtige Orientierungsprozesse bringen könnten. Diese Sichtweise verstellt jedoch den Blick auf vergrabene Organisationsstrategien sozialer Bewegungen, die in der BRD kaum Beachtung finden, deren genauere Betrachtung sich aber durchaus lohnt.

,Organizing ist ein Sammelbegriff für eine unüberschaubare Vielfalt an Organisationsansätzen aus der US-amerikanischen Geschichte. Das zentrale Organizing-Versprechen lautet: Es bringt Menschen zusammen und organisiert sie, bietet ihnen Auswege aus der eigenen Ohnmacht, erhöht ihren Einfluss auf die eigene Lebensrealität und politisiert, demokratisiert und verändert - ausgehend von den alltäglichen Lebenszusammenhängen - die Gesellschaft (Rubin/Rubin 2008). Unter diesem Begriff politischer Basisorganisierung versammeln sich revolutionäre Organisationsansätze wie der militante Arbeiterbewegung Ende des 19. Jahrhunderts, der Arbeitslosenbewegung der 1930er Jahre oder des Black Power und Feminist Movement in den 1960er und 70er Jahren. Es zählen jedoch auch (neo-)liberale Gegenstrategien der Herrschaftssicherung dazu.

Die US-amerikanische Organizing-Debatte untersucht dieses Spannungsfeld zwischen revolutionärer (transformativer) und herrschaftssichernder (liberaler) Politik von unten. Aus diesen Diskussionen kommen oftmals treffsichere Analysen staatlicher und unternehmerischer Strategien der Vereinnahmung und Repression von progressiven Basisbewegungen. Gleichzeitig verweisen viele Beiträge der Debatte auf kritische Aspekte linker Mobilisierungsstrategien, wenn diese beispielsweise statt der sozialen Frage eher subkulturelle Milieugrenzen bearbeiten. Und schließlich setzt die Organizing-Debatte das Handeln linker sozialer Bewegungen ins Verhältnis zu den Strategien der Herrschaftssicherung und versucht revolutionäre Auswege zu finden.

Die Besonderheit revolutionärer Organizing-Praxis liegt darin, dass sie die scheinbare linke Grundsatzentscheidung zwischen träger Organisation und dynamischer Bewegung aufbricht und dadurch einen dynamischen Begriff der Basisorganisierung anbieten kann. Der jahrzehntelange Aktivist und anarchistische Organizer Chris Crass kritisiert mit Blick auf den WTO-Gipfel in Seattle 1999, dass die öffentliche Diskussion nach den Protesten einen ,autonomen Mythos“ kreiert hätte.[2] Dieser Mythos verkläre militante soziale 
Proteste zu spontaner und unorganisierter Wut, zu deren ,Organisation“ ein Aufruf, eine Website, Flyer und der Startpunkt einer Demonstration ausreichen würden. Dabei seien die Proteste in Seattle Ergebnis jahrelanger Basisorganisierung (ebd.).

Die Debatte um das Für und Wider großer (Basis-)Organisationen ist natürlich älter als der WTO-Gipfel 1999. Sie wird seit über hundert Jahren in politischen Strategie-, aber auch in wissenschaftlichen Debatten geführt. Spätestens seit Roberto Michels aus der Bürokratisierung der deutschen Sozialdemokratie ein „ehernes Gesetz Oligarchie“ ableitete (Michels 1911), das eine quasi-naturgesetzliche Verknöcherung und Ent-Demokratisierung jeglicher Organisierungsbemühungen unterstellte, verläuft diese Debatte entlang der Konjunkturwellen politischer Diskurse. Viele Autor_innen verweisen auf die Fallstricke, wenn „Poor People“s Movements“ Organisierung um der Organisation willen betreiben, anstatt gesellschaftliche Veränderungen anzustreben (Piven/Cloward 1979). Wir schließen uns politischen (Williams/ Awatramani 2015) und wissenschaftlichen (Piven/Cloward 1979; Clemens/ Minkoff 2004) Einschätzungen an, die diese organisatorischen Tücken nicht als Zwangsläufigkeit, kollektive Organisierung aber als Notwendigkeit betrachten. Es erscheint uns plausibel, dass linke Bewegungen die Verhältnisse nur ins Wanken bringen können, wenn sie Organisationsformen finden, in denen sich massenhaft Betroffene verbindlich, langfristig und demokratisch organisieren können. Dass diese Formen auch bei zunehmender Größe basisdemokratisch und taktisch variabel bleiben können, das zeigen weltweit viele Beispiele, und wir trauen es den Beteiligten zu.

\section{Dynamische Basisorganisierung - alles fordern, viel gewinnen!}

Verbindliche und schlagkräftige Basisorganisationen sollten im 21. Jahrhundert als notwendiges Gegenstück zu wellenhaften Protesten auf der Straße gesehen werden. Abseits von Protestkonjunkturen können sie linke Politik greifbar machen und sozial verankern, ihre Basis verbreitern und Interessierte politisch und praktisch ausbilden. Wie der kommunistische Widerstandskämpfer Sebastian Franck schon 1938 feststellte, ist Organisierung eine

„[...] Notwendigkeit für die proletarische Klasse, weil nur sie dem zentralen Kampfapparat des bürgerlichen Staates eine Gegenmacht gegenüberstellen kann, weil nur sie wirksam die Vereinzelung und Machtlosigkeit des Proletariers aufhebt und weil das organisatorische Prinzip des geringsten Kraftaufwandes, der möglichsten Kraftersparnis und damit Kraftsteigerung Voraussetzung der Machtentfaltung ist.” (Franck 1972: 58)

Entsprechende Organisationen sollen gesellschaftliche Konflikte „bis an ihre äußeren Grenzen treiben” (Piven/Cloward 1979: 91, Übers. d. A.), um alles zu gewinnen, was zum jeweiligen Zeitpunkt möglich ist (ebd.: 37).

Transformative beziehungsweise revolutionäre Basisorganisationen fordern alles (Williams 2013), gewinnen viel (Mann 2011) und sind Trägerinnen von Bewegungen, die von gesellschaftlich marginalisierten Menschen gemacht wird. Sie sind mit „herzzerreißenden Situationen“ (Mann, zit. n. 
Maruschke 2014: 19) konfrontiert, wenn beispielsweise Menschen unter erzwungener Illegalität ins Land gebracht werden und in Konkurrenz zu anderen Niedriglöhner_innen treten. Ihre Arbeit liegt in gesellschaftlichen Feldern, in denen das solidarische Miteinander und eine klare Strategie die allgemeinen Zumutungen überbrücken müssen. Wie aber kann eine klare Vorstellung von Organisierung und Mobilisierung aussehen?

\section{Basisarbeit - das Herzstück des Organizings}

Das „Labor and Community Strategy Center“ ist die aktuell größte und schillerndste revolutionäre Basisorganisation in den USA. Ausgehend von jahrelangen gewerkschaftlichen Kämpfen gegen General Motors in den 1980er Jahren (Mann 2001), in denen sich tausende Arbeiter_innen in Fabrik und Nachbarschaft erfolgreich gegen GM organisiert haben, wurde das „Strategy Center" 1989 in Los Angeles gegründet. Im Schatten des Hollywoodglamours, umgeben von einer Arbeitslosenquote von 22 Prozent und knapp 40.000 Obdachlosen (US Census Bureau 2014), betreibt es mittlerweile ein eigenes Organizing-Ausbildungszentrum, einen Verlag, eine Radiosendung und zwei unabhängige Basisorganisationen: die „Bus Riders Union“ (BRU) und die „Community Rights Campaign“(CRC). Allein die BRU hat 3.00o Mitglieder, 30.00o feste Unterstützer_innen und monatliche Vollversammlungen, an denen 300 Menschen teilnehmen.

Bei der Betrachtung der Arbeit des „Strategy Centers“ werden einige Grundzüge erfolgreicher Basisorganisierung deutlich. Das Herzstück eines jeden Versuchs, langfristig und verbindlich immer mehr Menschen zusammenzubringen, ist die politische Arbeit mit ihnen und der Aufbau persönlicher Beziehungen. So banal es klingen mag, ohne Respekt vor anderen Lebenssituationen, ohne Geduld, ohne Offenheit und Interesse für kulturelle Unterschiede, ohne die Menschen in ihrer Widersprüchlichkeit zu mögen, gelingt keine Basisorganisierung. Die Herausforderung besteht darin, diese Selbstverständlichkeiten in eine alltägliche Praxis zu übersetzen. Sind wir bereit, voneinander auch Persönliches zu lernen, bewusst dafür zu sorgen, dass ein Treffen kein kaltes Abklopfen der Tagesordnungspunkte von MultiAktivist_innen, sondern ein Ort ist, an dem auch Menschen ohne linken Polit-Habitus gerne zusammen kommen?

Hier setzt transformatives Organizing an. Es bietet Menschen, die im institutionalisierten Politikbetrieb keine Lobby haben, die mit wenig Privilegien ausgestattet sind, die ausgebeutet und diskriminiert werden, die an der „Frontlinie des Neoliberalismus“"(Goldberg 2008) stehen, die Möglichkeit des gemeinsamen Kampfes für bessere Arbeits- und Lebensbedingungen. Es soll diejenigen erreichen, ,die von den rechten Angriffen ohne Hoffnung oder Gemeinschaftlichkeit zurückgelassen wurden“" (Mann, zit. n. Maruschke 2014: 19).

\section{Vom Kontakt zur Organisierung}

Wollen sich Menschen gemeinsam organisieren, müssen sie in Kontakt kommen. Die BRU des „Strategy Centers“ geht jeden Tag mit Festangestellten und Freiwilligen durch den öffentlichen Nahverkehr in Los Angeles und spricht die Menschen in den ,Fabriken auf Rädern' konkret an. Andere Organisationen 
gehen durch Arbeitsämter oder in der Nachbarschaft von Tür zu Tür. Die aufwendige Kontaktaufnahme bringt den Organisationsprozess nur weiter, wenn das erste Gespräch nicht abstrakt bleibt, sondern konkretes Interesse am politischen Projekt geweckt wird und Kontaktmöglichkeiten ausgetauscht werden.

Ist dieser erste Schritt gelungen, dann beginnt die eigentliche Arbeit einer Basisorganisation. Die Interessierten sollten so persönlich wie möglich eingeladen werden. Ein regelmäßiger Anruf vor dem nächsten großen Treffen, ein offenes Ohr für die persönlichen Anliegen und die Meinung des Gegenübers machen es Interessierten viel einfacher, sich einzubringen. Zwei Faustregeln des Organizings erleichtern die Arbeit: (1) Rede nicht mehr als dein_e Gesprächspartner_in; (2) Menschen müssen drei Mal persönlich kontaktiert werden, bevor sie zu einem Treffen kommen.

Von diesem Punkt an stellen sich neue Fragen: Verstehen Menschen, die wahrscheinlich zum ersten Mal in einer politischen Gruppe zusammenarbeiten, wann, wie und wo Entscheidungen getroffen werden? Gibt es verschiedene Angebote, sich einzubringen je nach Zeitbudget und Motivation? Sind die Verantwortlichkeiten klar definiert? Werden interessierte Menschen regelmäßig eingeladen und kontaktiert? Sind die Treffen auch für Alleinerziehende attraktiv, oder anders ausgedrückt: Habt ihr Respekt vor der Zeit der Leute, fangt ihr pünktlich an und hört ihr pünktlich auf?

Ist auch dies geglückt, stellt sich die Frage, wie die Problemlagen Einzelner zur politischen Auseinandersetzung verallgemeinert und mit anderen Kämpfen verbunden werden können. Menschen für ein politisches Projekt zu begeistern, das den engen, vom Eigeninteresse vorgegebenen Rahmen verlässt, ist eine langfristige Angelegenheit, für die ein langer Atem, Beharrlichkeit und eine stetige Radikalisierung notwendig sind.

Gesellschaftliche Gruppen sind trotz objektiv gleicher Interessenlagen weder homogen zusammengesetzt noch in ihren Lebensrealitäten widerspruchsfrei. Bei dem Versuch gemeinsamer politischer Arbeit weisen historische und aktuelle Erfahrungen auf die dringende Notwendigkeit hin, dass „politische Bildung ein wesentlicher Teil von Nachbarschaftsorganisierung sein [muss]“ (Fisher 1994: 227f., Übers. d.A.). Politische Bildung verstehen wir als ein strukturiertes gemeinschaftliches Nachdenken über unsere Alltagserfahrungen, die dabei in einen größeren Zusammenhang von Diskriminierung, Ausbeutung und Unterdrückung gestellt werden. Als Ergebnis erhoffen wir uns, dass die Beteiligten die Schuld an eigener Armut nicht bei sich selbst oder anderen Prekären suchen, sondern in gesellschaftlichen Strukturen.

Politische Bildungsarbeit fordert die Beteiligten auch emotional heraus. Es ist einfacher gesagt als getan, gemeinsam Vorurteile zu verlernen, Unterwerfungen zu benennen, Konkurrenzen zu überdenken und sich letztlich in einem kollektiven Prozess selbst zu verändern. Wir glauben, dass wir gestärkt aus solchen Prozessen herauskommen und lebhafte Analysen formulieren können, in denen sich Menschen mit ähnlichen Erfahrungen wiederfinden.

\section{Die Weisheit der Gruppe}

Die Anerkennung unterschiedlicher Lebensrealitäten ist bei diesem Unterfangen der Ausgangspunkt, um Gemeinsamkeiten auf dem Arbeitsmarkt, in der Konfrontation mit polizeilicher Repression oder bei anderen Problemen 
zu erkennen. Bildungsprozesse von unten können eine neue kollektive Stärke hervorbringen und beugen reaktionären Entwicklungen vor, die bei zahlreichen scheinbar ,unpolitischen ' Organizing-Projekten festzustellen war. Ist Bildungsarbeit fest verankerter Teil der eigenen Praxis, wird durch regelmäßige Aufarbeitung der eigenen Lebensrealitäten die Gesellschaftsanalyse präzisiert es entsteht eine neue Sicht auf die Welt. Die „Weisheit der Gruppe“ (Williams 2013, Übers. d. A.) wird so zur Grundlage von Entscheidungen für oder gegen Kampagnen, für oder gegen Bündnisse und für die gezielte Ausbildung von Organizer_innen beziehungsweise Aktivist_innen.

Neben dieser breiter angelegten, gemeinschaftlichen politischen Bildung haben viele transformative Organisationen in den USA Ausbildungszentren oder Lehrgänge für Menschen geschaffen, die mehr Verantwortung in der politischen Arbeit übernehmen wollen. Viele soziale Bewegungen flachten auch deshalb ab, weil die inhaltliche und praktische Ausbildung dem Zufall überlassen wurde. Dies führte zu einer Dominanz privilegierter Schichten in den organisatorischen Zusammenhängen, die aus einem materiell gesicherten Umfeld viel Zeit und erlernte Fähigkeiten in die Bewegung einbringen können. Wenn diese wenigen Protagonisten die Bewegung verlassen, ist solch eine Lücke dann kaum zu schließen.

Das „Strategy Center“ hat aus dieser historischen Erfahrung gelernt und bildet seit mehr als zwei Jahrzehnten inhaltlich und praktisch aus. Inzwischen stammt ein Großteil der festangestellten Frauen aus den armen und diskriminierten Nachbarschaften in Los Angeles. Sie werden professionell geschult und müssen die jahrzehntelang erprobten Techniken und Analysen nicht neu erfinden, sondern lediglich einem Realitätscheck unterziehen. Der erste Tag jedes neuen Mitglieds in der „National School for Strategic Organizing“ findet in den Bussen von Los Angeles statt.

Für eine ähnlich starke Verankerung praktisch-politischer Schulungen von unten fehlt es hierzulande an Ressourcen und oftmals auch am Konsens über deren Priorität. Einige Gruppen betonen zwar zu Recht den weiterbildenden Effekt der alltäglichen Arbeit. Die praktische Erfahrung, fremde Menschen zum Jobcenter ins Herz der europäischen Niedriglohnhölle zu begleiten, sich auf sie wirklich einzulassen, sollte nicht unterschätzt werden. Für eine sich immer weiter verbreiternde widerständige Praxis muss Bildungsarbeit innerhalb der Gruppen und zwischen denselben institutionalisiert werden.

\section{Vom Einzelkonflikt zur Gesellschaft}

Kontinuierliche und gegenseitige Selbst- und Weiterbildung hilft den Einzelnen und der Gruppe, scheinbar individuelle Konflikte zu politischen Fragen der ganzen Gesellschaft werden zu lassen. Wie diese Fragen und die eigenen Forderungen in die Öffentlichkeit getragen und durchgesetzt werden können, muss in einer nachvollziehbaren, zugänglichen, demokratischen und für eine große Anzahl an Menschen geeigneten Form geschehen. Es müssen Diskussions- und Entscheidungsformen gefunden werden, die die Fallstricke einer übermäßigen Formalisierung und Bürokratisierung auf der einen Seite und der „Tyrannei der Strukturlosigkeit“ (Freeman 2013) auf der anderen umschiffen. Uns erscheinen hier weder komplexe, tagelange und mehrstufige Abstimmungsverfahren auf der Grundlage einer seitenlangen Satzung 
praktikabel, noch endlose Plenen, auf denen die letzten drei Anwesenden die Entscheidungen treffen.

Die BRU wählt regelmäßig einen Rat aus ihren Mitgliedern, die alltägliche Entscheidungen mit den Festangestellten Organizer_innen abstimmen. Auf monatlichen Vollversammlungen werden dann verbindliche, grundsätzliche Entscheidungen getroffen. Dazwischen führen die Organizer_innen möglichst viele Einzelgespräche mit den Mitgliedern, so genannte one-onones, um Meinungen einzuholen. Diese Gespräche werden ausgewertet, die Meinungen festgehalten und so in gemeinschaftliche Prozesse integriert.

Die entstehenden Kampagnen sollten, darin sind sich transformative Organisationen in den USA einig, einen Blick in die Welt ermöglichen, die erkämpft werden soll. Dabei ist es selbstverständlich, dass keine Kampagne zu hundert Prozent erfolgreich sein kann. Gleichzeitig sollte der durch die Kampagne aufgebrochene Konflikt, in dem Kampagnen nur Zwischenziele erreichen können, langfristig betrachtet werden. In Los Angeles gibt es nur deshalb ein flächendeckendes Nahverkehrssystem, weil die BRU der Stadt Mehrinvestition in Höhe von 2,7 Milliarden Dollar abgerungen hat. Die Basis und die Festangestellten der BRU haben dafür monatelang diskutiert und Forderungen und Vorgehen abgewogen und sind in einen Prozess gekommen, in dem sich die Einzelnen als Teil eines politischen Projekts sehen, das über die Bearbeitung von Einzelinteressen hinaus geht.

Mittlerweile ruft die BRU den „Fight for the Souls of our Cities“ aus und fordert unter anderem ein autofreies Los Angeles. Williams bringt diese Herangehensweise auf den Punkt, wenn er sagt:

„Wenn wir nur Kampagnen machen, die wir erfolgreich abschließen können, dann kämpfen wir aus meiner Erfahrung nicht für das, was unsere Leute verdient haben. Zweitens: Es gibt Zeiten, in denen die Angriffe von rechts mit so einer Intensität kommen, dass es sein kann, dass wir verlieren. Aber oft sind Menschen nicht notwendigerweise durch die Perspektive des Sieges, sondern dadurch inspiriert, dass wir ehrlich, in ihrem Interesse, auf ihre Art kämpfen. Und manchmal ist es einfach wichtig zu kämpfen, selbst wenn du weißt, dass sie dir die Zähne eintreten.“(Williams, zit. n. Maruschke 2014: 67)

Eine auf diese Weise verankerte Basisorganisation kann ein wichtiger Eckpfeiler sozialer Bewegungen sein. Dieser Zusammenhang bestätigt sich schon bei einem oberflächlichen Blick auf den Widerstand gegen Atommülltransporte im Wendland oder auch bei genauerem Hinsehen in der Occupy-Bewegung in den USA.

Denn entgegen dem angesprochenen, autonomen Mythos' war die OccupyBewegung in Oakland nicht nur wegen guter Websiten oder der Spontanität der Beteiligten schlagkräftig, sondern auch wegen dutzender beteiligter Basisorganisationen. Auf die gegenseitige Abhängigkeit von lokaler Verankerung und dynamischer Bewegungs- und Aktionsformen weist die transformative Organizing-Debatte schon seit einiger Zeit hin (Fisher/Shragge 2000). Auch in der BRD scheint sich diese Sicht langsam durchzusetzen (Schaber 2015).

\section{Eine visionäre Bewegung aufbauen...}

Es mangelt transformativen Basisorganisationen nicht an Schlagworten, die den politischen Gegner beschreiben. Eine eigene, vermittelbare 
gesellschaftliche Vision, die radikal über gegenwärtige Krisenmitverwaltung hinaus geht, an den jeweiligen Wurzeln der Alltagsprobleme ansetzt und in der Lage ist, die oftmals vereinzelten Kämpfe zu vereinen, wird jedoch noch nicht formuliert. Die Erfahrungen aus der tagtäglichen Arbeit zeigen aber, dass eine formulierte Utopie Menschen für eine Organisierung über den persönlichen Horizont hinaus begeistern kann. Die Alternative muss dabei in wenigen Sätzen vermittelbar sein und in der Praxis erprobt werden. Sie sollte verständlich, konkret und klar formuliert sein und sich nicht hinter subkultureller und akademischer Sprache verstecken.

Die Frage, in welcher Gesellschaft wir eigentlich leben wollen, ist nicht leicht zu beantworten. Eine ehrliche Debatte darüber ist nur in einer Atmosphäre möglich, welche die Praxis unterschiedlicher Organisationsansätze und ihre wechselseitige Ergänzung als Notwendigkeit erkennt. Eine solche Debatte sollte vom „Respekt für politischen und ideologischen Pluralismus“ (Lee et al. 2014, Übers. d. A.) getragen werden. Neben einer revolutionären Analyse von unten, politischer Basisarbeit und konfrontativen Politikformen ist eine solidarische Haltung zwischen den beteiligten Menschen und Organisationen unerlässlich. Die revolutionäre Kraft sozialer Bewegungen wird sich nicht zuletzt in einer „erneuerten linken Kultur“ (Lee et al. 2014, Übers. d. A.) ausdrücken.

\section{...um zu gewinnen!}

Basisorganisierung verlängert den politischen Zeithorizont und damit die Bewertungskriterien von Erfolg oder Misserfolg linker Politik. Ihre Stärke liegt nicht nur in kurzfristiger Mobilisierung, sondern im langfristigen Aufbau von Gegenmacht, verankert im Leben vieler Menschen. Im Ergebnis erscheinen soziale Bewegungen nicht mehr als phasenhaft aufflackernder Widerstand, sondern als Ergebnis jahrzehntelanger Organisierung entlang gesellschaftlicher Widersprüche, in welcher abseits der Hochphasen des Protests die Bewegung verbreitert wurde. So können in den Druckphasen der Bewegung die Konflikte bis an ihre äußersten Grenzen getrieben werden. Dafür sollten viele Gewohnheiten des linksradikalen Aktivismus und der institutionalisierten Linken in der BRD überdacht werden. Basisorganisierung braucht andere Formen und Räume als allein ein wöchentliches Plenum. Es muss genug Zeit geben, sich kennenzulernen, soziale Beziehungen aufzubauen und gemeinsames Vertrauen zu entwickeln. Dies können traditionelle große linke Institutionen aufgrund ihrer gegenwärtigen organisatorischen Trägheit und kleine Basisorganisationen vor Ort wegen fehlender Ressourcen praktisch kaum leisten. Flächendeckende und unbürokratische Basisorganisierung ist nicht ausschließlich in der Freizeit und als Hobby umsetzbar. Mit Blick in die US-amerikanische und südeuropäische Praxis müssen beide Seiten gemeinsam eine Lösung finden. Eine finanzielle Förderung als sinnvolle Möglichkeit zu diskutieren ist dabei die Aufgabe der einen Seite, sie unkompliziert bereitzustellen, die der anderen.

Die US-amerikanische Praxis des Transformativen Organizings eröffnet allen Interessierten eine strukturierte und jahrzehntelang reflektierte Herangehensweise an funktionierende und schlagkräftige Basisorganisierung, die revolutionäre Politik in der Gesellschaft verankert und greifbar werden lässt. Unabhängig von gesellschaftlichen Unterschieden innerhalb kapitalistischer Staaten 
und von den Fachbegriffen des Organizings verändert Basisorganisierung die politische Landschaft. Auf dem langen Weg der Veränderung der gesellschaftlichen Verhältnisse scheint dies dringend notwendig.

\section{Endnoten}

[1] Zwei Beispiele aus der jüngeren Vergangenheit: Die dreiteilige Workshopreihe zum Thema Basisorganisierung auf dem BUKO-Kongress 2014 oder die vom „Bündnis Zwangsräumungen verhindern“ organisierte Konferenz im Sommer 2015 mit Basisorganisationen aus Spanien, Italien, der BRD u. a.

[2] Vortrag von Chris Crass (2010): Presentation of the Catalyst Organizing Project in Phoenix/Arizona. San Francisco.

\section{Autor_innen}

Claudia Kratzsch ist Aktivistin in den Bereichen Erwerbslosigkeit und Migration.

Robert Maruschke ist Politikwissenschaftler und arbeitet zu Bürgerbeteiligung, Organizing und sozialen Protesten.

\section{Literatur}

Clemens, Elisabeth S. / Minkoff, Debra C. (2004): Beyond the iron law. Rethinking the place of organizations in social movement research. In: David A. Snow / Sarah Soule / Hanspeter Kriesi (Hg.), The Blackwell companion to social movements. Malden u. a.: Blackwell, 155-170.

Fisher, Robert (1994): Let the people decide. Neighborhood Organizing in America. New York: Twayne Publishing.

Fisher, Robert / Shragge, Eric (2000): Challenging community organizing. Facing the 21st century. In: Journal of Community Practice 8/3, 1-19.

Franck, Sebastian (1972 [1938]): Zur Kritik der politischen Moral. Ein Beitrag zur Konzeption einer neuen sozialistischen Bewegung. Giessen: Prolit-Buchvertrieb.

Freeman, Jo (2013 [1972]): The tyranny of structurelessness. In: WSQ: Women's Studies Quarterly 41/3-4, 231-246.

Goldberg, Harmony (2008): Building power in the city. Reflections on the emergence of the Right to the City Alliance and the National Domestic Workers Alliance. https://inthemiddleofthewhirlwind.wordpress.com/building-power-in-the-city/ (letzter Zugriff am 17.3.2015).

Lee, N’Tanya / Munoz, Cinthya / Poblet, Maria / Warren-White, Josh / Williams, Steve (2014): Towards a Transformational Strategy. https://leftroots.net/towards-strategy/ (letzter Zugriff am 17.3.2015).

Mann, Eric (2001): A race struggle, a class struggle, a womens struggle all at once. Organizing on the Buses of LA. In: Socialist Register 37/37, 259-273.

Mann, Eric (2011): Playbook for Progressives 16 Qualities of the Successful Organizer. Boston: Beacon Press.

Maruschke, Robert (2014): Community Organizing. Zwischen Revolution und Herrschaftssicherung. Eine kritische Einführung. Münster: edition assemblage.

Michels, Robert (1911): Zur Soziologie des Parteiwesens in der modernen Demokratie. Leipzig: Werner.

Piven, Frances Fox / Cloward, Richard A. (1979): Poor People's Movements. Why They Succeed, How They Fail. New York: Vintage.

Rubin, Herbert J. / Rubin, Irene (2008): Community Organizing and Development. Boston: Allyn \& Bacon. 
Schaber, Peter (2015): Gewalt! In: Neues Deutschland. http://www.neues-deutschland.de/ artikel/965349.gewalt.html (letzter Zugriff am 24.3.2015).

US Census Bureau (2014): Los Angeles Quick Facts. http://quickfacts.census.gov/qfd/ states/o6/o64400o.html (letzter Zugriff am 24.3.2015).

Williams, Steve (2013): Fordert Alles! Lehren aus dem Transformativem Organizing. New York: Rosa-Luxemburg-Stiftung. http://www.rosalux-nyc.org/wp-content/files_mf/ williams_transformatives_organizing.pdf (letzter Zugriff am 12.11.2013).

Williams, Steve / Awatramani, Rishi (2015): New Working-Class Organization and the Social Movement Left. In: Socialist Register 51/51, 1-15.

\section{Grassroots Organizing changes the political landscape}

Contemporary discussions from within leftist social movements put much emphasis on organizational questions. This essay criticizes the popular thesis of activists and social movement scholars according to which dynamic social movements and solid organizations contradict each other. Instead, the authors call for an interlocked understanding of movements and organization. They outline a few critical aspects of leftist grassroots organizing and run a reality check. Are dominant strategies of social movements able to invite people to actively take part in social conflicts? Can people join together to work on a vision of our society that is not confined to day-to-day struggles? The concept of Transformative Organizing and a dialogue with German initiatives guide the authors on their quest. 